Revue bibliographique pour le domaine irano-aryen

\title{
Elspeth R. M. Dusinberre. Empire, Authority, and Autonomy in Achaemenid Anatolia
}

\section{Astrid Nunn}

\section{(2) OpenEdition}

1 Journals

\section{Édition électronique}

URL : http://journals.openedition.org/abstractairanica/45526

DOI : 10.4000/abstractairanica.45526

ISBN : 1961-960X

ISSN : 1961-960X

Éditeur :

CNRS (UMR 7528 Mondes iraniens et indiens), Éditions de l'IFRI

Référence électronique

Astrid Nunn, "Elspeth R. M. Dusinberre. Empire, Authority, and Autonomy in Achaemenid Anatolia », Abstracta Iranica [En ligne], Volume 37-38-39 | 2018, document 49, mis en ligne le 30 décembre 2018, consulté le 26 septembre 2020. URL : http://journals.openedition.org/abstractairanica/45526 ; DOI : https://doi.org/10.4000/abstractairanica.45526

Ce document a été généré automatiquement le 26 septembre 2020

Tous droits réservés 


\title{
Elspeth R. M. Dusinberre. Empire, Authority, and Autonomy in Achaemenid Anatolia
}

\author{
Astrid Nunn
}

\section{RÉFÉRENCE}

Elspeth R. M. Dusinberre. Empire, Authority, and Autonomy in Achaemenid Anatolia. Cambridge-New York: Cambridge University Press, 2013. p. xxvi, 374p. (paperback reprint 2015).

1 L'A. est une spécialiste de l'Anatolie achéménide et offre, après sa monographie sur Sardes (2003), une synthèse élargie à toute l'Anatolie. Celle-ci resta sous contrôle perse achéménide de 547 à 334 av. J.-C. Bien qu'il existe déjà de nombreuses recherches sur l'Anatolie achéménide, d'une part le matériel archéologique de cette époque augmente chaque année et d'autre part, notre approche étant toujours influencée par son temps, change. Pendant un temps le modèle du «centre et périphérie » a prédominé pour expliquer la politique, l'acculturation etc. d'empires étendus. De même « the tempered sovereignty, ... which involves the notion that certain nations or regions might operate with some, often circumscribed, sovereignty of various sorts within an overarching sovereign state » (p. 3) est trop chargée de notions que chacun interprète à sa manière. Ainsi, l'axe de recherche de l'A. est dorénavant le modèle " authority-autonomy » qui permet plus de flexibilité dans l'analyse des variations complexes de la relation entre dominants et dominés.

Cette monographie est ordonnée selon des chapitres thématiques, avec, sous-jacente, la question de savoir ce que la domination achéménide a apporté et/ou changé. Les sources utilisées sont inégalement réparties et aussi bien épigraphiques qu'archéologiques. L'A introduit tout d'abord les paysages géographiques très différents de cette région (Chapitre 1). Les thèmes du chapitre 2 couvrent 
l'administration (satrapes, satrapies, taxes, routes, palais. Les peintures de Altintepe sont bien urartéennes: A. Nunn, "Wandmalerei in Urartu », in: S. Kroll, C. Gruber, U. Hellwag, M. Roaf, P. Zimansky (Hg.), Biainili-Urartu, 2007, (Acta Iranica 51), p. 321-337. Dans ce même chapitre l'A. aborde les archives, les langues parlées et écrites ainsi que l'iconographie des sceaux et des monnaies. Selon l'A. leur iconographie montre combien l'emprise perse est profonde. Certes cette emprise existe; mais j'aimerais évoquer un aspect qui pourrait la relativiser. Le motif du héros est très fréquent. Même si sa signification générale est évidente, celle dans son contexte internationalisé achéménide l'est bien moins. Symbolise-t-il vraiment le pouvoir perse ? À mon avis, il n'est pas certain que ce héros d'antique origine mésopotamienne soit lié à l'idée royale. Je pencherais plutôt vers une idée abstraite de force liée à la protection, telle celle qui émane des nombreuses figures protectrices du genre laHmu. Ceci nous montrerait qu'une fois de plus la forme choisie pour une image est étrangère mais que le contenu en est local (L'A cite Nunn 1996, qui correspond à ma thèse non publiée et non diffusée. Voir la version quelque peu modifiée et publiée = A. Nunn, Der figürliche Motivschatz, OBO SA 13, 2000).

3 Le chapitre 3 est dédié au contrôle politique et militaire analysé par le biais des textes et des fortifications (Sardes, Dascyleion, Meydancıkkale e.a). L'A. ayant inclus l'iconographie dans le chapitre précédent, on se demande pourquoi la tombe de Tatarlı avec la représentation de soldats manque dans celui-ci. Les scènes de banquet, les coupes et rhytons en métal ou terre sont assez nombreux pour justifier un chapitre (4) sur «Eating and drinking ». L'A. insiste à raison sur l'importance du banquet dans l'antiquité et tout particulièrement en Anatolie achéménide. Dans ce domaine aussi il est très difficile de faire la part entre fond et forme. Le chapitre 5 est dédié au culte funéraire. Les tombes livrent un des marqueurs les plus passionnants. Elles sont nombreuses et témoignent d'une variété presque illimitée, englobant les traditions locales, mais aussi grecques et orientales. Sans témoin écrit ou recherche poussée ces tombes ne disent rien sur les défunts, d'autant moins que les objets funéraires sont très standardisés. En passant, le manque de discussion sur de nombreux points litigieux est peut-être inévitable dans une synthèse. L'interprétation des défunts dans la nécropole de Deve Hüyük comme soldats d'une garnison perse est beaucoup moins sûre que l'A. ne le présente (p. 104, 184-187) (A. Nunn, Nekropolen und Gräber in Phönizien, Syrien und Jordanien zur Achämenidenzeit, Ugarit-Forschungen 32, 2000, 407-408). Ainsi se pose-t-on la question de savoir si, sans études approfondie, les tombes reflètent vraiment la devise « identity, authority and autonomy " (p. 206). "Worshipping the Divine » est le titre du prochain chapitre 6. De la Phrygie à Sardes et Xanthos, les images de dieux sont bien différentes. Elles démontrent la continuation d'anciens dieux (Kubaba, Cybèle, Tarkunt ou Malija...) mais aussi l'apparition de nouveaux, ou même plus fréquemment, la reprise de dieux autochtones sous d'autres formes. Suivent deux chapitres sur l'éducation et l'identité achéménide en Anatolie.

4 Les illustrations suffisent pour bien comprendre le texte. Celui-ci offre une bonne synthèse accessible à tous. Mais les questions si difficiles - relation entre forme (de la mode) et fond (de la culture), entre absorption volontaire et indépendante du régime politique et adaptation à une élite (perse) ...-, qui éclaireraient ce patchwork qu'était l'Anatolie achéménide, sont à peine posées. Il aurait suffi de le faire et de remettre leur réponse à plus tard. 


\section{AUTEURS}

\section{ASTRID NUNN}

Université de Munich 